\title{
MODELO DE CASCADA DE ATENCIÓN PARA LA CO-INFECCIÓN DE LAS HEPATITIS VIRALES PARA EL CUIDADO CONTINUO EN PERSONAS CON VIH/SIDA
}

\section{Care Continuum Model for Coinfection of Viral Hepatitis for the Continuing Care in people living with HIV / AIDS}

\author{
Leandro Tapiaa, Paola Peña ${ }^{b}$, Laura Manosalvas ${ }^{c}$ y Robert Paulino-Ramírez ${ }^{d}$
}

Recibido: 10 de marzo, 2019 • Aprobado: 8 de junio 2019

Cómo citar: Tapia L, Peña P, Manosalvas L, Paulino-Ramírez R. Modelo de Cascada de Atención para la co-infección de las hepatitis virales para el cuidado continuo en personas con VIH/Sida. cysa [Internet]. 25 de noviembre de 2019 [citado 27 de noviembre de 2019];3(3):35-1. Disponible en: https://revistas.intec.edu.do/index.php/cisa/article/view/1542

\section{Resumen}

Introducción: las coinfecciones con hepatitis virales y el VIH representan un riesgo por su alta transmisibilidad y complicaciones. Es de vital importancia que se puedan identificar las barreras de acceso y manejo de las coinfecciones del VIH y la Hepatitis B y C. El propósito del estudio es describir la cascada de atención para la coinfección de las hepatitis virales en personas viviendo con VIH/SIDA.

Métodos: se delimitaron los indicadores de manejo y seguimiento de los pacientes monitoreados actualmente en clínicas de primer nivel de atención en Santo Domingo y Santiago. Los datos recopilados fueron analizados a partir de los registros escritos de los pacientes en seguimiento, para evaluar los datos epidemiológicos y serológicos que se obtuvieron de los archivos clínicos.

Resultados: se demostró que un $100 \%$ de los pacientes fueron enrolados, $85.7 \%$ se consideraron como retenidos en el manejo de la coinfección VIH/VHB y solamente

\footnotetext{
a Instituto de Medicina Tropical \& Salud Global, Universidad Iberoamericana, Santo Domingo, República Dominicana

Centro de Orientación e Investigación Integral, Santo Domingo, República Dominicana. Correo-e: 1.tapia@prof.unibe.edu.do

b Instituto de Medicina Tropical \& Salud Global, Universidad Iberoamericana, Santo Domingo, República Dominicana.

Correo-e: paolaipena@gmail.com
}

\begin{abstract}
Introduction: Co-infections of viral hepatitis and HIV represent a risk due to their high transmissibility and complications. It is vitally important that barriers to access and management of HIV and Hepatitis B and C co-infections can be identified. The purpose of the study is to describe the cascade of attention for the preparation of viral hepatitis in people living with HIV/AIDS.

Methods: The management and follow-up indicators of the patients currently monitored in first-level care clinics in Santo Domingo and Santiago were delimited. The data collected was analyzed from the written records of the patients in follow-up to evaluate the epidemiological and serological data that was obtained from the clinical files.

Results: It was demonstrated that $100 \%$ of the patients were enrolled, $85.7 \%$ were considered as retained in care of HIV / HBV coinfection and only $71.4 \%$ received ARTbased on Tenofovir. The cascade of care for HIV / HCV

\footnotetext{
${ }^{\mathrm{c}}$ Instituto de Medicina Tropical \& Salud Global, Universidad Iberoamericana, Santo Domingo, República Dominicana.

Correo-e: lauramanosalvase@gmail.com

d Instituto de Medicina Tropical \& Salud Global, Universidad Iberoamericana, Santo Domingo, República Dominicana

Centro de Orientación e Investigación Integral, Santo Domingo, República Dominicana. Correo-e: r.paulino1@unibe.edu.do
} 
$71.4 \%$ recibieron TARV basado en Tenofovir. La cascada de atención para la coinfección VIH/VHC muestra un vínculo de $87.5 \%$ de los pacientes, $75 \%$ fueron retenidos y ninguno recibió tratamiento.

Conclusión: la identificación de los factores de riesgo que influyen en las brechas de la cascada es fundamental para optimizar el manejo y monitoreo de los pacientes coinfectados, teniendo en cuenta que es preciso poder reconocer aquellos infectados para iniciar tratamiento de inmediato y así prever secuelas citopatológicas hepáticas.

Palabras claves: cascada de atención; VIH; VHB; VHC; coinfección.

\section{Introducción}

La Organización de las Naciones Unidas (ONU) el 1 de enero del 2016 puso en acción los "17 Objetivos para Transformar Nuestro Mundo" dentro de la Agenda 2030 para el Desarrollo Sostenible, en donde el objetivo 3.3 busca que para el 2030 se elimine la epidemia del Síndrome de Inmunodeficiencia Adquirida (SIDA) y se combatan las hepatitis virales $^{1,2}$.

El Virus de Inmunodeficiencia Humana (VIH) es un virus de la familia retroviridae que se propaga a través de los fluidos corporales y ataca el sistema inmune del cuerpo, específicamente las células $\mathrm{CD} 4+$ o células $\mathrm{T}^{3}$. Este es el virus que puede llevar al Síndrome de Inmunodeficiencia Adquirida (SIDA) si no es tratado ${ }^{4}$. Según la Organización Mundial de la Salud en el 2017 había un estimado de 36,900,000 personas infectadas con el virus del VIH en el mundo, mientras que en América se estimó que 3,400,000 personas estaban infectadas con el virus ${ }^{5}$. En la República Dominicana la Organización Mundial de la Salud (OMS) estima que hay alrededor de 68,000 personas que estaban infectadas con el mismo virus ${ }^{6}$.

Actualmente, a nivel mundial, existen 257,000,000 de personas con infección por el VHB y un $0.7 \%$ de coinfection shows a linkage to care of $87.5 \%$ of patients, $75 \%$ were retained and none received treatment.

Conclusions: Identifying the risk factors that influence the gaps in the cascade of care is essential to optimize the management and monitoring of coinfected patients, with special interest in those that might receive immediate treatment to prevent liver cytopathological sequelae.

Keywords: Cascade of care; HIV; HBV; HCV; coinfection.

la población en América se encuentra infectado7. La infección aguda por el VHB se establece por la presencia del antígeno superficial $(\mathrm{HBs} A g)$ y de la inmunoglobulina $\mathrm{M}$ en el antígeno del núcleo $(\mathrm{HBcAg})^{8}$. Sin embargo, la infección crónica es determinada por la presencia de $\mathrm{HBsAg}$ por más de seis meses ${ }^{8}$. La OMS recomienda la administración de tratamiento con tenofovir ya que son los fármacos más potentes y efectivos para suprimir la replicación del $\mathrm{VHB}^{7}$. La vacuna contra el VHB tiene una eficacia del $95 \%$ para la prevención del mismo ${ }^{7}$. Las poblaciones más susceptibles a padecer la infección por el VHB son grupos vulnerables a la exclusión social como los inmigrantes, UD y $\mathrm{HSH}^{9}$.

Se estima que en Latinoamérica hay más de 12,000,000 de casos de Hepatitis por infección del VHC y en el Caribe una prevalencia de $1.5 \%{ }^{10}$. El diagnóstico de la infección por el VHC se hace mediante la detección de anticuerpos anti-VHC con una prueba serológica y se confirma con la detección del ácido ribonucleico del virus ${ }^{11}$.

La cura por el VHC se logra mediante el uso de un antiviral de actuación directa (AAD) de segunda generación o una combinación de un AAD de primera generación co-administrada con interferon y ribavirin ${ }^{11}$. Estos esquemas incluyen: simeprevir, sofosbuvir, daclatasvir, combinación de ledipasvir y 
sofosbuvir, combinación de sofosbuvir y velpatasvir, combinación de sofosbuvir, velpatasvir y voxilaprevir, combinación de glecaprevir y pibrentasvir. Estos esquemas se toman entre 8 a 48 semanas, donde el paciente ya empieza a presentar mejoría ${ }^{12-14}$.

Las poblaciones vulnerables, que tienen más riesgos de infección por VHC incluyen: inmigrantes, usuarios de drogas endovenosas (UD), trabajadores sexuales, $\mathrm{HSH}$ y personas afectadas por el $\mathrm{VIH}^{10}$.

La dimensión de la infección por VHB y VHC en la República Dominicana no está bien caracterizada, y los datos al momento de esta revisión eran inespecíficos.

Actualmente, entre el 5 y el $20 \%$ de los pacientes $\mathrm{VIH}+$ presentan la coinfección con el VHB ${ }^{12}$. Para el tratamiento de esta coinfección se recomienda que a estos pacientes se les suministre emtricitabina, lamuvidina, tenofovirdisoproxilfumarato $y / o$ incluíralafenamida de tenofovir ya que tienen acción contra VIH y VHB ${ }^{13}$. A nivel mundial se estima que 2.3 millones de personas están coinfectadas con el VHC y VIH ${ }^{14}$. La recomendación actual para el tratamiento de esta coinfección es el régimen inicial de antirretrovirales, sin embargo, este debe ser elegido cuidadosamente debido a las interacciones entre los medicamentos y las toxicidades de estos ${ }^{13}$.

Las cascadas de atención son estructuras para el seguimiento y monitoreo de los pacientes que padecen de una enfermedad, detallando la progresión de esta desde el diagnóstico y el tratamiento hasta el seguimiento de los mismos ${ }^{15}$. Para hacer una cascada de atención con pacientes que padecen de la infección por el VHB se propone evaluar los siguientes criterios: a) identificar pacientes con demostrada infección VHB, b) analizar la proporción de pacientes con un diagnóstico de infección por VHB que esté ligada a algún centro de salud, c) analizar la proporción de pacientes que reciben manejo para la infección de VHB, y d) identificar la proporción de pacientes que acuden a sus consultas de seguimiento y determinar la proporción de pacientes que recibieron terapia antiviral siguiendo las guías basadas en evidencia para el manejo de paciente con infección $\mathrm{VHB}^{16}$. La cascada de atención para VHC debe describir: el tamizaje de anticuerpos contra VHC, pacientes enrolados y retenidos en el sistema de salud, así como aquellos en tratamiento ${ }^{17}$.

Esta investigación se realizó con el fin de proponer un modelo de monitoreo de las coinfecciones $\mathrm{VIH} / \mathrm{VHB}$ y VIH/VHC, cuantificar los pacientes retenidos, los pacientes enrolados, los pacientes con tratamiento antiviral y los pacientes que lograron una supresión viral en los centros de atención primaria para el VIH/SIDA. Otro de los objetivos de este modelo de cascada de atención, es caracterizar las barreras de retención y seguimiento en poblaciones de riesgo y su impacto en el futuro de la transmisión de las hepatitis virales.

\section{Metodología}

Los criterios de selección de los participantes estuvieron basados en la evidencia registrada de infección por VIH/SIDA y co-infección con VHB y/o VHC, y que acudieron a seguimiento de VIH/ SIDA durante un período de 8 años, de enero 2009 a diciembre 2017, en dos centros de atención primaria en las provincias de Santo Domingo y Santiago, respectivamente. La muestra de estudio fue seleccionada de forma no probabilística por conveniencia. Los participantes seleccionados debieron tener más de 18 años de edad y mantener un seguimiento en el Centro de Orientación e Investigación Integral (COIN) de Santo Domingo y el Hospital Periférico Juan XXIII en Santiago de los Caballeros. Se consideró un participante como "enlazado a los servicios" cuando se pudo confirmar con pruebas de laboratorio la infección por VIH. Se identificó como "retenido" a todos los pacientes que acudieron 
a una o más visitas cada 6 meses durante su enfermedad. Se clasificó "en tratamiento" a todos los pacientes recibiendo terapia farmacológica pertinente a su infección, y como "curado" a todo paciente cuyo síntomas y signos, al igual que sus pruebas diagnósticas, demostraron la finalización de su infección.

El análisis por grupo de riesgo se estableció por la auto identificación de hombre que tiene sexo con otros hombres (HSH), identidad de género trans (TG), reporte de uso de drogas los últimos 12 meses (UD), y trabajo sexual (TRSX).

El estudio fue realizado con la aprobación del Comité de Ética Institucional de la Universidad Iberoamericana (UNIBE) bajo el código CEI2017-160E y de los centros COIN y Hospital Periférico Juan XXIII para ser llevada a cabo. Los resultados fueron analizados utilizando el software de análisis estadísticos STATA $^{\mathrm{TM}}$ ver.15.0.

\section{Resultados}

Un total de 2,787 pacientes cumplían con el criterio de infección por VIH al momento del estudio, de los cuales 352 asisten al Hospital Periférico Juan XXIII y 2,435 al COIN. Sus expedientes clínicos fueron revisados para confirmar dicha infección; de estos, 36 participantes tenían evidencia de resultados de laboratorio de co-infección VIH/VHB ( $\mathrm{n}=28)$, VIH/ VHC ( $\mathrm{n}=8$ y VIH/VHB/VHC $(\mathrm{n}=1)$.

Se encontró que de las personas con una prueba positiva del virus de hepatitis B, el $100 \%$ fue enlazado a los servicios de salud; el $85.7 \%$ fue clasificado como retenido; mientras que el $71.4 \%$ recibió TARV basada en Tenofovir (Anexos, figura 1).

La cascada de atención de la coinfección VIH/VHC en este estudio muestra que el $87.5 \%$ de los pacientes fue enrolado al cuidado y manejo de su condición, mientras que un $85.7 \%$ de estos se retuvo en el cui- dado. Ningún paciente recibió tratamiento para esta coinfección por lo que no pudieron llegar a la cura (Anexos, figura 2).

La cascada de atención para los pacientes usuarios de drogas y HSH con la coinfección VIH/VHB: se encontró que todos los diagnosticados con esta coinfección estaban enrolados en los servicios de salud, y de estos, un $85 \%$ fueron retenidas, mientras que un $75 \%$ recibieron TARV basada en Tenofovir (Anexos, figura 1).

Respecto a la cascada de atención para para los pacientes usuarios de drogas y $\mathrm{HSH}$ con coinfección VIH y Hepatitis C, encontramos que todos los diagnosticados estaban enrolados, y un $75 \%$ de estos, retenidos; sin embargo, ninguno de los pacientes recibió tratamiento para la Hepatitis $\mathrm{C}$ (Anexos, figura 2).

El análisis de correlación de Pearson en la población estudiada no arrojó ninguna correlación bivariable entre el estatus de VHB y VHC entre los grupos de riesgo, y que pudiese ser considerado como significativo.

\section{Discusión}

Este estudio evidencia que existen barreras para la adherencia en el cuidado de los pacientes coinfectados con el VIH y VHB, ya que solamente un $86 \%$ de los pacientes se pudieron adherir al programa de seguimiento de su co-infección VIH/VHB. Asimismo, existen barreras en la obtención de la terapia antiviral óptima ya que un $29 \%$ de los pacientes coinfectados no está en esta terapia.

En cuanto al modelo de cascada de atención para la co-infección VIH y VHC, se observó de igual forma elementos que podrían impedir que $25 \%$ de los pacientes coinfectados se adhieran al cuidado. En los centros estudiados, ningún paciente pudo 
recibir terapia para la infección crónica del VHC, por lo que no lograron una progresión satisfactoria por la cascada de atención.

Para las poblaciones en riesgo podemos ver que un $15 \%$ de los pacientes coinfectados con VIH y VHB no está adherido al cuidado, mientras que un $25 \%$ de estos no recibe su terapia adecuada, por lo que pueden representar un foco infeccioso para ambos virus ya que pertenecen a una demografía con una prevalencia más elevada a la población general.

Debido a que los últimos estudios revelan la probabilidad de curar la infección por Hepatitis C, uno de los pasos necesarios para poder lograr la completa eliminación y posterior erradicación de la misma, es conocer la dimensión exacta de la situación de la infección en la República Dominicana, y lograr que para el 2030 se pueda eliminar su transmisión.

Para la co-infección del VIH y VHC en poblaciones de alto riesgo podemos observar una barrera en cuanto a la obtención de los medicamentos apropiados, ya que ninguno de estos ha recibido tratamientos para su co-infección. Estos hallazgos sugieren la necesidad de estudios de tipo cualitativos que puedan identificar las barreras de atención en cada una de las poblaciones de riesgo y en aquellas que no tienen elementos de riesgo identificados para caracterizar las intervenciones necesarias en la prestación de servicios. En estudios realizados de la infección por VIH, exclusivamente, se han podido identificar que elementos como estigma, discriminación basada en género y orientación sexual, ausencia de servicios comprensivos, entre otros, sirven como barreras de retención en los servicios de salud. ${ }^{18,19,20,21}$

Recomendamos enfatizar en la realización de las pruebas diagnósticas de hepatitis virales, así como estratificar las infecciones por genotipificación y cuantificación viral para poder establecer los estadios de las infecciones y ofrecer un inicio de trata- miento farmacológico oportuno. Esto último en una época en que podemos eliminar la transmisión de la hepatitis $\mathrm{C}$ por tratamiento farmacológico y evitar las nuevas infecciones por hepatitis $\mathrm{B}$ a través de la vacunación intensiva en poblaciones de alto riesgo es un momento único en la salud global.

\section{Bibliografía}

1. Organización de las Naciones Unidas. Resolución de la Asamblea General de las Naciones Unidas. Transformar nuestro mundo: la Agenda 2030 para el Resolución Desarrollo Sostenible. [21 de octubre de 2015]; consultado el 8 de agosto de 2017. Disponible en: http://www.un.org/ga/search/ view_doc.asp?symbol=A/RES/70/1\&referer $=/$ english/\&Lang=S

2. United Nations. (2019). Good health and well-being. The Sustainable Development Goals Report The Sustainable Development Goals Report 2019, 26-29. doi: 10.18356/179bc73d-en

3. Murray PR, Rosenthal KS, Pfaller MA. Microbiología Médica. Barcelona: Elsevier; 2014.

4. World Health Organization. (2016). Consolidated guidelines on the use of antiretroviral drugs for treating and preventing Hiv infection (Vol. 2). Retrieved from https://www.who.int/ hiv/pub/arv/arv-2016/en/

5. World Health Organization. Number of people (allages) living with HIV Estimates by WHO region. GHO | Bycategory | Number of people (Allages) living with HIV - Estimatesby WHO region. [August, 02 2019; consultado el 18 de julio de 2017]. Available from http://apps.who. int/gho/data/view.main.22100WHO?lang=en (Number of people (allages) living with HIV Estimatesby country. GHO | Bycategory | Number of people (Allages) living with HIV - Estimatesby country. http://apps.who.int/gho/data/node. main.620?lang=en 
6. Organización Mundial de la Salud 2017. Hepatitis B. [19 de julio de 2019; consultado el 18 de julio de 2017]. Available from http://www. who.int/mediacentre/factsheets/fs204/es/

7. Centers for Disease Control and Prevention 2018. Viral Hepatitis. Hepatitis B Questions and Answers for Health Professionals. [October 8, 2019; accessed October 6, 2018] Available from https://www.cdc.gov/hepatitis/hbv/hbvfaq.htm\#general ().

8. Jose-Abrego A, Panduro A, Fierro NA, Roman $S$. High prevalence of $\mathrm{HBV}$ infection, detection of subgenotypes F1b, A2, and D4, and differential risk factors among Mexican risk populations with low socioeconomic status. J Med Virol. 2017;89: 2149-57.

9. Petruzziello A, Marigliano S, Loquercio G, Cozzolino A, Cacciapuoti C. Global epidemiology of hepatitis $C$ virus infection: An up-date of the distribution and circulation of hepatitis C virus genotypes. World J Gastroenterol. 2016;22: 7824-40.

10. Bessone F, Brahm J, Bustios C, Cheinquer H, Contreras F, Díaz G, et al. Recomendaciones para el manejo de la Hepatitis C en América Latina. (1era. ed.) Lima: Asociación Latinoamericana para el estudio del Hígado (ALEH); 2018.

11. NHS. Hepatitis C Treatment. [June 21, 2018; accessed October 22, 2018]. Available from https://www.nhs.uk/conditions/hepatitis-c/ treatment/

12. HIV/AIDS JUNPo, editor. UNAIDS. Global AIDS Update. Geneva: United Nations; 2016.

13. Ledesma J, Jimenez M, Caballero T, Adames R, et al. Guía de Atención de las Hepatitis Virales y sus Comorbilidades asociadas al VIH. (1era. ed.) Ministerio de Salud Pública; Junio 2018.
14. WorldHealthOrganization. More than 2 million people coinfected with HIV and hepatitis C. [March 7 2016; accessed October 6, 2018]. Available from http://www.who.int/hiv/mediacentre/news/hep-hiv-coinfected/en/

15. Evashwick CJ. Creating a continuum. Thegoalis to provide an integrated system of care. HealthProg.1989;70: 36-9, 56

16. McMahon BJ. Editorial Commentary: Sliding Down the Cascade of Care for Chronic Hepatitis B Virus Infection. ClinInfectDis. 2016;63: 1209-11.

17. Viner K, Kuncio D, Newbern EC, Johnson CC. The continuum of hepatitis $\mathrm{C}$ testing and care. Hepatology. 2015;61: 783-9.

18. Kutnick AH, Gwadz MV, Cleland CM, et al. It's a Process: Reactions to HIV Diagnosis and Engagement in HIV Care among High-Risk Heterosexuals. Frontiers in PublicHealth. 2017;5: 100. doi:10.3389/fpubh.2017.00100

19. Hickey MD, Odeny TA, Petersen M, et al. Specification of implementation interventions to address the cascade of HIV care and treatment in resource-limited settings: a systematic review. ImplementationScience cience : IS. 2017;12: 102. doi:10.1186/s13012-017-06

20. Ayieko J, Brown L, Anthierens S, et al. "Hurd leson the path to 90-90-90 and beyond": Qualitative analysis of barriers to engagement in HIV care among individuals in rural East Africa in the context of test-and-treat. Costa AB, ed. PLoS ONE. 2018;13(8): e0202990. doi:10.1371/journal.pone.0202990.

21. Tapia L, Pena P, Manosalvas L, Camilo A, Routy JP, Paulino-Ramirez R. Improving continuum of care in dominican republic for hiv-infected patients co-infected with hepatitis b and c. Amer Jour of Trop Med and Hyg 2018 jan 1, vol. 99, no. 4, pp. 620-620. 

personas con VIH/SIDA

\section{Anexos}

Figura 1. Cascada de atención para la co-infección VIH y Virus de Hepatitis B.

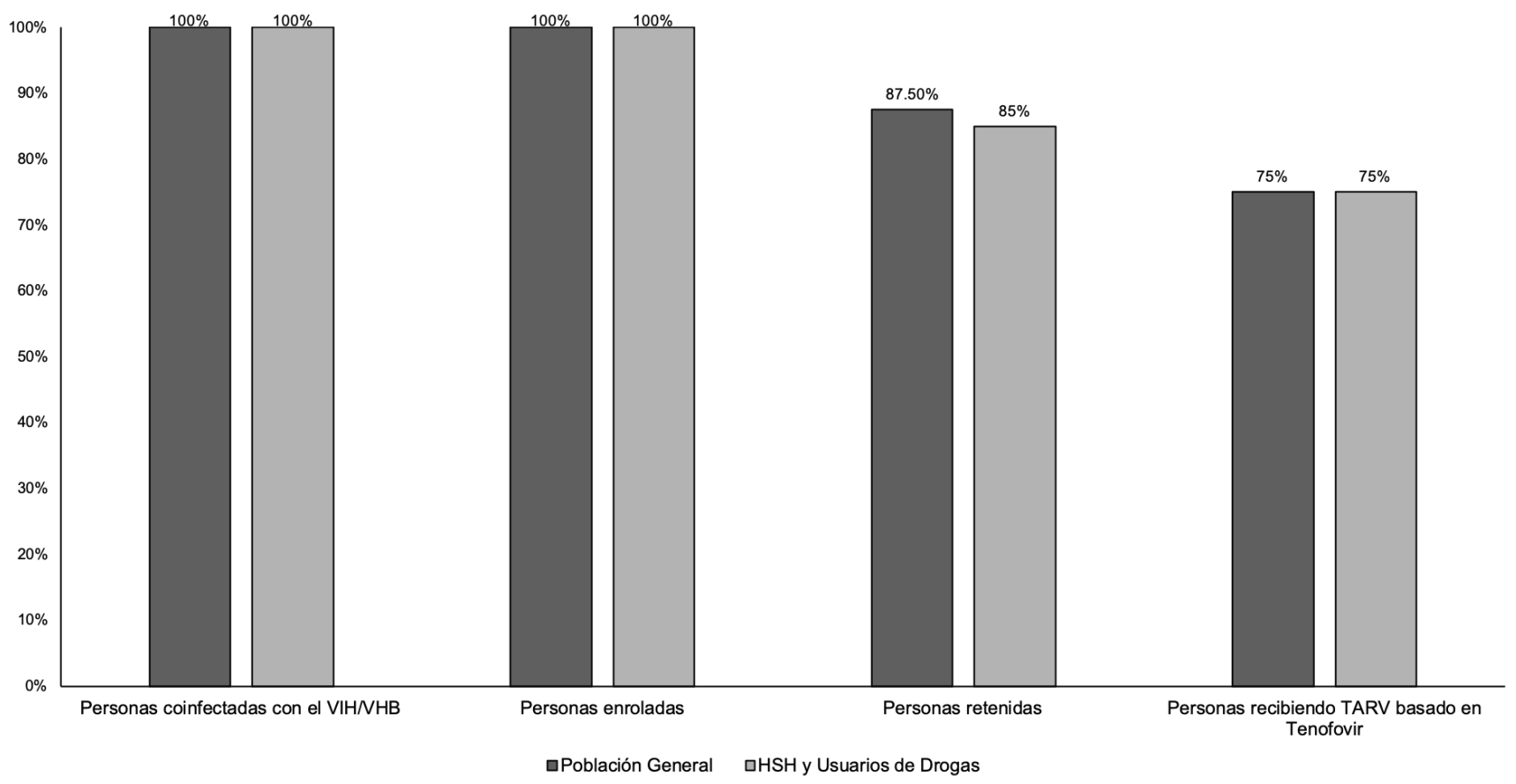

Figura 2. Cascada de atención para la co-infección VIH y Virus de Hepatitis C.

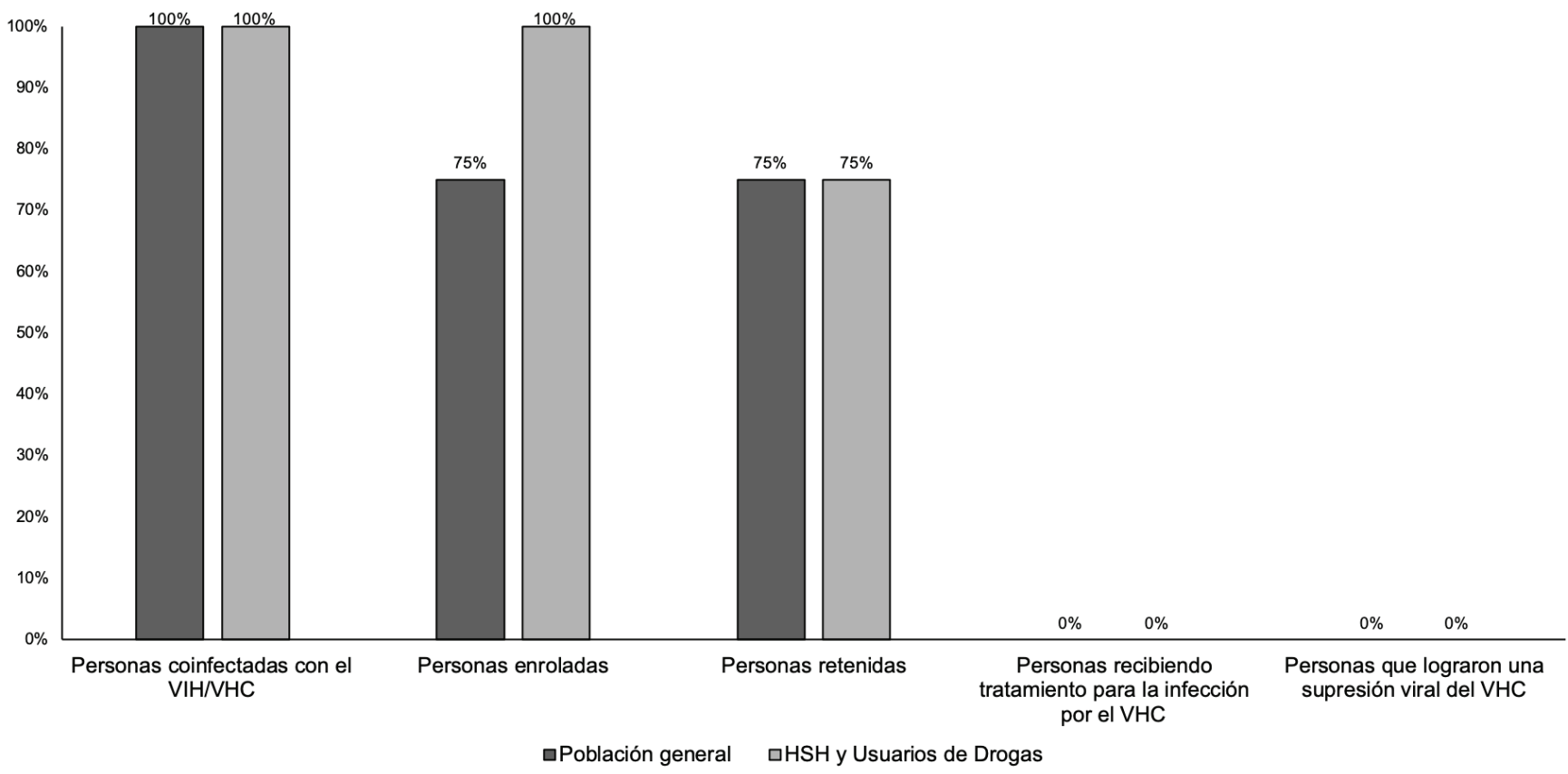

